\title{
Particle Transport and Stochastic Acceleration in the Giant Lobes of Centaurus A
}

Stephen O'Sullivan

Technological University Dublin, stephen.osullivan@tudublin.ie

Follow this and additional works at: https://arrow.tudublin.ie/scschmatcon

Part of the Astrophysics and Astronomy Commons

\section{Recommended Citation}

O'Sullivan, S. (2011). Particle transport and stochastic acceleration in the giant lobes of Centaurus A. AIP Conference Procedures, vol. 1367, no. 76. doi:10.1063/1.3628719 INTERNATIONAL SYMPOSIUM ON THE RECENT PROGRESS OF ULTRA-HIGH ENERGY COSMIC RAY OBSERVATION AIP Conf. Proc. 1367, 76 (2011) http://dx.doi.org/10.1063/1.3628719

This Conference Paper is brought to you for free and open access by the School of Mathematics at ARROW@TU Dublin. It has been accepted for inclusion in Conference papers by an authorized administrator of ARROW@TU Dublin. For more information, please contact arrow.admin@tudublin.ie, aisling.coyne@tudublin.ie,gerard.connolly@tudublin.ie.

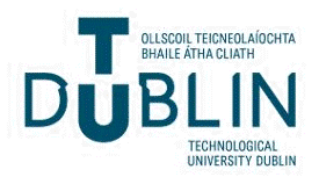




\title{
Particle transport and stochastic acceleration in the giant lobes of Centaurus A
}

\author{
Stephen O'Sullivan \\ School of Mathematical Sciences, Dublin Institute of Technology, Dublin, Ireland
}

\begin{abstract}
The conditions within the giant lobes of Centaurus A are reviewed in light of recent radio and $\gamma$-ray observations. Data from WMAP and ground-based telescopes in conjunction with measurements from Fermi-LAT constrain the characteristic field strength and the maximum electron energy. The implications for the transport of energetic particles are discussed in terms of residence times and cooling times within the lobes. Acceleration of electrons and UHECR via the second order Fermi mechanism is discussed.
\end{abstract}

Keywords: cosmic ray origin, radiogalaxies, gamma-ray sources (astronomical), acceleration, magnetic fields PACS: $98.54 . \mathrm{Gr}, 98.70 . \mathrm{Sa}$

\section{INTRODUCTION}

Cosmic rays with energies in excess $3 \mathrm{EeV}$, those above the so-called ankle in the cosmic ray flux, will be referred to as ultra-high energy cosmic rays (UHECRs). The origins of these particles remain an open question. However, there has been convincing agreement between experiments including Auger [1, 2], HiRes [3, 4], on the existence of a GZK cutoff at $\sim 50 \mathrm{EeV}$.

For proton UHECR (pUHECR), this cutoff is consistent with stochastic energy loss due to highly inelastic photo-meson interactions $\left(p+\gamma_{\mathrm{CMB}} \rightarrow p / n+\pi^{0 /+}\right)$ which is expected to be the dominant loss mechanism for energies above $\sim 40 \mathrm{EeV}$. At similar energies, nuclei UHECR (nUHECR) will fragment into lighter particles. Hence the process of photodisintegration $\left((\mathrm{A}, \mathrm{Z}) \gamma_{\mathrm{CMB} / \mathrm{CIB}} \rightarrow\left(\mathrm{A}^{\prime}, \mathrm{Z}^{\prime}\right)+\left(\mathrm{Z}-\mathrm{Z}^{\prime}\right) p+\right.$ $\left.\left(\mathrm{A}-\mathrm{A}^{\prime}+\mathrm{Z}-\mathrm{Z}^{\prime}\right) n\right)$ for nUHECR interacting with CMBCIB photons will result in a GZK suppression at similar energies to those described for pUHECR undergoing photo-meson interactions. The propagation losses in either case will limit the GZK horizon for $\sim 50 \mathrm{EeV}$ UHECR to $\sim 100 \mathrm{Mpc}$.

Within this $100 \mathrm{Mpc}$ horizon, there are a limited number of classes of object which are viable accelerators. In large part, this is due to the requirement on any accelerator to have a sufficiently large magnetic field to constrain particles during the acceleration process and furthermore, a sufficiently weak photon field such that losses due to synchrotron and photo-meson energy losses are not large. Amongst the rather limited number of object classes which satisfy these criteria are radio-loud and -quiet AGNs, high and low luminosity GRBs, magnetars, and galaxy clusters.

With such a restrictions on the range of possible sources, the issue of correlating UHECR with objects might appear to be a potentially straightforward matter. However, given the low statistics available to date, interpretation of the data has been fraught with difficulty. In particular, the interpretations of data from Auger and HiRes in this respect have not been complementary, although it should be stressed, probably not contradictory either.

An early study by the Auger Collaboration [5] of the 27 Auger events available with over $57 \mathrm{EeV}$ showed a high degree of correlation with AGN inside a $75 \mathrm{Mpc}$ radius from the Véron-Cetty and Véron catalogue. While the level of significance of the correlation fell somewhat in a subsequent study [6], there is still a strong indication that UHECR follow some structure in the universe which is coincident with the distribution of AGN. Of particular note is a strong association of events with Cen A: 12 out of 58 observed high-energy events were found to be within $18^{\circ}$. However, it must be noted that Cen A lies directly in front of the Centaurus cluster which is itself part of the Hydra-Centaurus supercluster. Complicating interpretation of these data however, HiRes does not appear to support this association of high energy events with large-scale structure [7].

The question of composition must temper any discussion on correlating detections with sources. With considerable dependence on the hadronic interaction model used, the characteristics of the showers resulting from UHECR may be used to infer composition. It has been suggested that Auger sees a UHECR spectrum which is primarily light nuclei or protons below $\sim 10 \mathrm{EeV}$ and intermediate to heavy nuclei above this value $[8,9]$. Conversely, it has been suggested that observations from HiRes indicate lighter composition at the highest observed energies $[10,11]$.

If the inference made from the Auger data is correct, and UHECR demonstrate a real bias towards heavy com- 
position, there is not just an apparent stress with the HiRes analysis, but also an internal stress with the Auger data itself. Approximately rectilinear propagation may be plausible for protons propagating through the galactic and extra-galactic magnetic fields. On the other hand, without a detailed knowledge of these fields, the deflections suffered by heavier nuclei will mean the loss of any possible association with sources $[12,13,14]$.

Regardless of the possibly spurious nature of any direct association with Auger UHECR detections, Cen A is nevertheless a promising candidate for an acceleration site of UHECR. Cen A the closest FR I source at $\sim 3.4 \mathrm{Mpc}$ and as a consequence is well studied at all energies.

In the remainder of this paper, I shall first review the characteristics of the giant radio lobes (GLs) of Cen A based on observational data with particular emphasis on the deduction of the magnetic field strength and the maximum electron energy based on WMAP and groundbased radio observations as well as Fermi-LAT $\gamma$-ray observations. In the next section, the transport of energetic particles within the lobes is briefly discussed. Finally, the viability of stochastic acceleration as a channel for the replenishment of energetic electrons and generation of UHECR is addressed.

\section{CEN A GIANT LOBE EMISSION}

\section{Lobe characteristics}

The characteristic scale of the GLs of Cen A as observed in radio frequencies is of order $100 \mathrm{kpc}$ [15]. The standard picture of FR I sources is that of an uncollimated jet driving a subsonic expansion of material into the surrounding environment [16]. Perhaps due to a level of detail not observable in farther sources, Cen A does not quite fit this picture. In particular, within the southern GL there is the smaller scale $(\sim 5 \mathrm{kpc})$ southwest lobe defined by a surrounding shell of shocked material. Assuming pressure balance and an ISM gas temperature of $0.35 \mathrm{keV}$, Croston and et al. [17] estimate an expansion speed of $\sim 2500 \mathrm{kms}^{-1}$ (in agreement with figures derived by [15]). Taking this inferred rate of expansion as an upper bound for the GLs as well, a dynamical age of the GLs of order 100 Myr may be estimated. Similar figures may be arrived at through various other estimates [in particular, see 15,18$]$.

Strongly turbulent fields may prevail in radio lobes. There is observational evidence from polarization maps for chaotic fields in the southern lobe of Cen A [19]. Motivated by such arguments, a correlation length for the magnetic field within the GLs of $\mathrm{L}_{\mathrm{c}}=10 \mathrm{kpc}$ may be adopted. It is also assumed that equal energy exists in the mean and turbulent components of the field.

\section{Electron cooling}

The principal energy dissipation channels for high energy electrons are synchrotron and inverse-Compton (IC) radiative losses. Observations in radio to gamma-ray constrain models of acceleration by dictating energies and localities of relativistic electrons in GLs.

Synchrotron radiation is emitted from with a peak energy at a critical frequency $\left(v_{\text {synch }} / \mathrm{THz}\right) \approx$ $16(B \sin \theta / \mu \mathrm{G})(E / \mathrm{TeV})^{2} \propto B E^{2}$ where $\theta$ is the electron pitch angle [20] where $B$ is interpreted as the root mean square magnetic field. These energy losses define a corresponding synchrotron radiative lifetime $\left(\tau_{\text {synch }} / \mathrm{Myr}\right) \approx$ $33.5(B \sin \theta / \mu \mathrm{G})^{-\frac{3}{2}}\left(v_{\text {synch }} / \mathrm{THz}\right)^{-\frac{1}{2}} \propto B^{-2} E^{-1}$. Hence in a $1 \mu \mathrm{G}$ field for isotropically distributed pitch angles $\left(\left\langle\sin ^{2} \theta\right\rangle=2 / 3\right), \quad\left(v_{\text {synch }} / \mathrm{THz}\right) \approx 12.2(E / \mathrm{TeV})^{2}$ is obtained with a corresponding radiative lifetime of $\left(\tau_{\text {synch }} / \mathrm{Myr}\right) \approx 12.6(\mathrm{E} / \mathrm{TeV})^{-1}$.

IC radiation has a critical frequency $v_{\mathrm{IC}} \approx 4 \times$ $10^{12}(E / \mathrm{TeV})^{2} v_{\text {seed. }}$. Energy losses have the same dependency as synchrotron losses with a proportionality given by $U_{B} / U_{\mathrm{CMB}}$. For a $1 \mu \mathrm{G}$ background field and taking $U_{\mathrm{CMB}} \approx 0.25 \mathrm{eV} \mathrm{cm}^{-3}$ with an associated peak frequency $v_{\mathrm{CMB}}=160.2 \mathrm{GHz}, v_{\mathrm{IC}} \approx 640 \mathrm{ZHz} \approx$ $2.7 \mathrm{GeV} / h$ is found and the corresponding radiative lifetime $\left(\tau_{\mathrm{IC}} / \mathrm{Myr}\right) \approx 1.26(E / \mathrm{TeV})^{-1}$. Hence there is an order of magnitude dominance expected for $\gamma$-ray emission from IC radiation. As a result, the radiative lifetime incorporating the effects of both loss processes is given by $\left(\tau_{\text {cool }} / \mathrm{Myr}\right) \approx 1.15(E / \mathrm{TeV})^{-1}$.

\section{Proton cooling}

pUHECR within the lobes of Cen A will radiate energy primarily through synchrotron and $\mathrm{p}-\mathrm{p}$ interactions [21, 22]. The characteristic cooling times are $\left(\tau_{\text {synch }}^{\mathrm{p}} / \mathrm{Myr}\right) \approx 1.4 \times 10^{8}(E / \mathrm{EeV})^{-1}(B / \mu \mathrm{G})^{-2}$ and $\left(\tau_{\mathrm{pp}} / \mathrm{Myr}\right) \approx 1.7 \times 10^{6}\left(n_{\mathrm{p}} / 10^{-4} \mathrm{~cm}^{-3}\right)^{-1}$ respectively.

\section{Spectral ageing}

Observing synchrotron radiation, particularly at high frequencies such that a cutoff associated with spectral aging is measured, allows us to study the source electron energy distribution (EED). If the break extends farther than may be attributed to simple aging of the high energy component of the population, local particle reacceleration may offer an explanation . In particular, when 
stochastic turbulent acceleration is significant, the break will depend on factors influencing acceleration efficiency such as the turbulence level of the magnetic field rather than the age of the underlying plasma.

All of this discussion is subject to reservations due to the questionable reliability of spectral age inferences. Even in the case of FR II sources where dynamical ages are quite tightly constrained, the difficulties in obtaining agreement from spectral ageing models are well documented [23] with inferred values often being an order of magnitude lower than expected.

It is supposed that the principal energization of the electron population occurs at the hotspot in the case of FR II sources and at the core for FR I sources such as Cen A. As a consequence, the oldest plasma for FR II sources is expected at the base of the radio lobes where shock-processed exhaust gas remain from the earliest phase of jet propagation. This is broadly supported by observations of the break frequency in radio spectra rising with distance from the core in the case of FR II sources. The case for FR I sources is far less clear. Naively, it may be assumed that given the universal lack of a hotspot at the extremities of the lobes, the primary acceleration of the synchrotron emitting electron population is at the base of the outflow. However, if it is the case that FR I sources are merely powered-down FR II sources, the relationship between plasma age and distance from core is not clear.

In light of earlier discussions, the EED may be assumed to follow a broken power law $N(E) \propto E^{-p}$ where [eg. 24]

$$
p=\left\{\begin{array}{ll}
2 \alpha_{\mathrm{in}}+1 & \text { for } E_{\mathrm{min}} \leq E<E_{\mathrm{br}} \\
a \alpha_{\mathrm{br}}+b & \text { for } E_{\mathrm{c}}<E
\end{array} .\right.
$$

While adiabatic expansion is important, it alters the emission frequency of all electrons by a uniform factor and hence changes the frequency and amplitude scaling of the spectrum but not the functional form. The form itself depends on the degree of tangling in the field, the efficacy of pitch-angle scattering, and the injection process.

For the continuous injection (CI) model [25], which assumes a power law of electrons is continuously pumped into the radio source, these parameters are $a=1, b=1 / 2$. Spectra in diffuse regions often steepen more rapidly than described by CI suggesting that the stock of high energy electrons is not as effectively replenished as provided for by the model.

Models which follow a single burst injection of a power law are often more suitable in regions where the cutoff is faster than described by CI. In the KardashevPacholczyk (KP) model [26, 25], electrons maintain the same pitch angle with respect to the $\mathrm{B}$ lines and lose energy according to $a=4 / 3, b=1$.

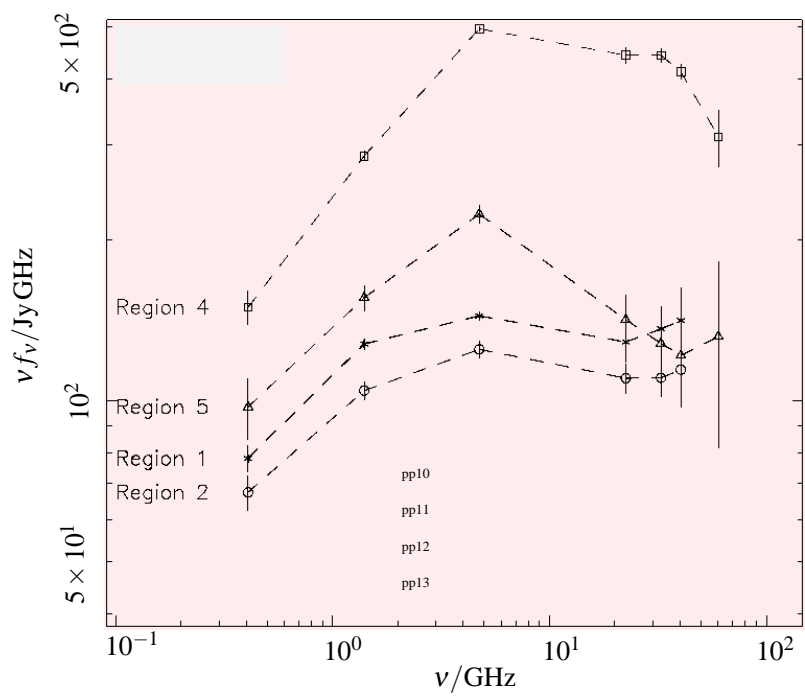

FIGURE 1. Flux density $\times$ frequency $\left(v f_{v}\right)$. Northern lobe: outer (Region 1) and inner (Region 2) components. Southern giant lobe: inner (Region 4) and outer (Region 5) components. Reproduced from [22] by kind permission of the authors.

The Jaffe-Perola (JP) is a single burst model [27] permits permanent pitch angle isotropization which leads to an exponential steepening (not described by the above equation) for the high-frequency spectrum.

\section{WMAP}

Hardcastle and et al. [22] analysed five-year Wilkinson Microwave Anisotropy Probe (WMAP) data of Cen A. The spectral energy densities (SEDs) for the lobes show breaks at $5-20 \mathrm{GHz}$ for the southern lobe and $1-5 \mathrm{GHz}$ for the northern lobe. Figure 1 presents a panel of a figure from [22] which shows these breaks in the SEDs for the outer and inner regions of each of the GLs of Cen A derived from both ground-based and satellite datasets. In particular, the outer and inner regions of the northern lobe (labelled Region 1 and Region 2 respectively) show no significant steepening of SED beyond the break whereas going from the inner to outer regions of the southern lobe (labelled Region 4 and Region 5 respectively) reveals a marked increase in the spectral index beyond the break. It is found that the southern lobe spectra are well fit by the JP model and furthermore suggested that this may be explained if the last injection of electrons now dominating the synchrotron radiation in this region took place around 30 Myr ago [22].

In the case of the northern lobe, the radio spectra are fit by appealing to a continuous injection broken powerlaw model for the EED which naturally suggests ongoing 


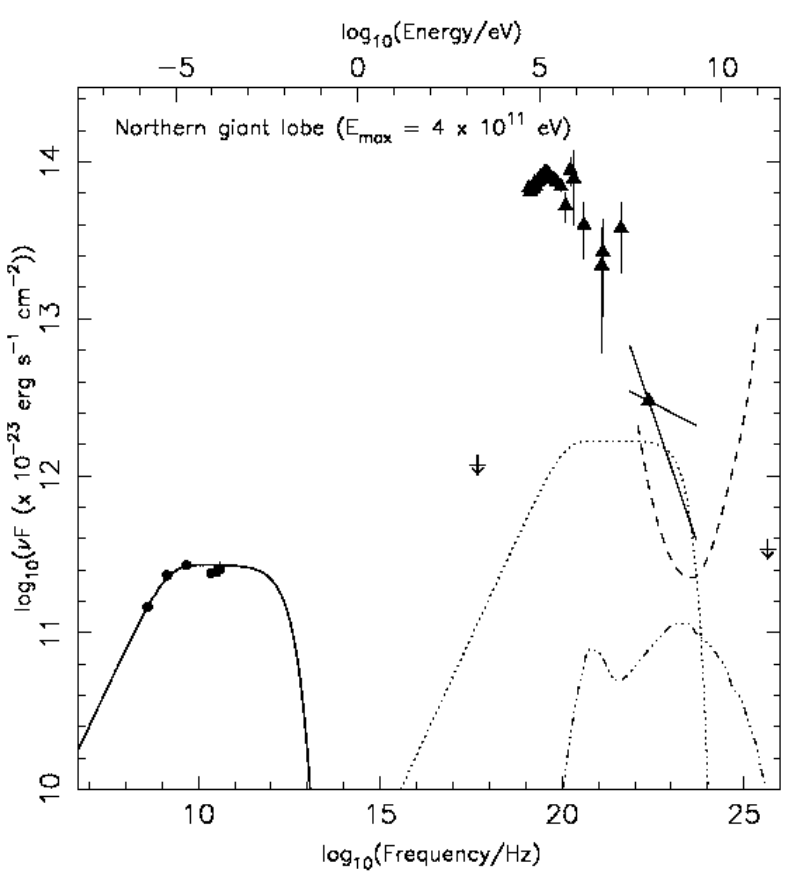

FIGURE 2. Predicted spectra for synchrotron (solid line) and inverse-Compton (CMB scattered: dotted line; EBL scattered: dot-dashed line) emission from northern GL of Cen A. The EED is assumed to conform to a a broken power-law with a cutoff at $0.4 \mathrm{TeV}$. Filled circles are combined WMAP and ground-based radio measurements. For details on remaining data points the reader is referred to [22]. This figure was reproduced from [22] by kind permission of the authors.

(or very recently ceased) replenishment of energetic electrons. A maximum energy of $0.4 \mathrm{TeV}$ is proposed by the authors as compatible with the model and observations. The predicted synchrotron and IC spectra for this case are shown in Figure 2.

\section{Fermi-LAT}

The Fermi Gamma-ray Space Telescope's Large Area Telescope (Fermi-LAT) has detected and imaged the radio lobes of Cen A in high energy $\gamma$-rays [28] (see Figure 3). The Fermi-LAT Collaboration has modelled this emission by parametrizing the electron energy distribution (EED) of with a broken power-law in the form:

$n_{e}=\left\{\begin{aligned} k_{e} \gamma^{s_{1}} & \text { for } \gamma_{\min } \leq \gamma<\gamma_{\mathrm{br}} \\ k_{e} \gamma_{\mathrm{br}}^{s_{2}-s_{1}} \gamma^{-s_{2}} \exp \left[-\gamma / \gamma_{\max }\right] & \text { for } \gamma \geq \gamma_{\mathrm{br}}\end{aligned}\right.$

Synchrotron continuum radio measurements are used to constrain all parameters of the model over a range in magnetic field strength $B=0.1 \mu \mathrm{G}$ to $B=10 \mu \mathrm{G}$. Given the EED, the IC radiation spectrum from scattering with the CMB and CIB may be derived. The best fit model

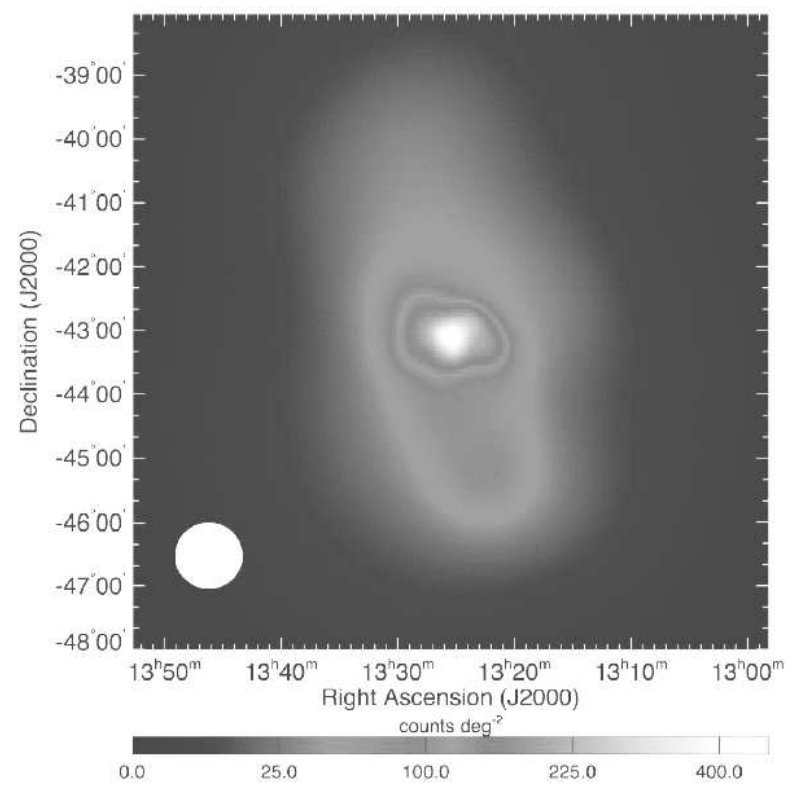

FIGURE 3. Fermi-LAT $\gamma$-ray $(>200 \mathrm{MeV})$ counts maps centered on Cen A. From [28]. Reprinted with permission from AAAS.

to the Fermi-LAT data indicates a maximum electron energy of $E_{e} \sim 0.1-1 \mathrm{TeV}$ and $B \sim 1 \mu \mathrm{G}$ (for example, see Figure 4 for fit to northern giant lobe's IC SED).

\section{TRANSPORT}

Understanding whether the high energy charged particles in the radio lobes of Cen $\mathrm{A}$ implied by observations are transported with sufficient rapidity to avoid heavy cooling losses, or whether there is re-acceleration at work compensating for these losses is clearly of importance.

This question must be addressed by considering whether highly relativistic particles may be efficiently directed from a remote acceleration region (eg. jet hotspot, black-hole) without significant energy losses.

For $B \sim 1 \mu \mathrm{G}$, the gyroradius is given by $r g \equiv$ $E /|Z| e B \approx 1.1 \times 10^{-6}|Z|^{-1}(E / \mathrm{TeV}) \mathrm{kpc}$. The rigidity in a field with $\mathrm{L}_{\mathrm{c}}=10 \mathrm{kpc}$ is given by $\rho \equiv r_{\mathrm{g}} / \mathrm{L}_{\mathrm{c}} \approx 1.1 \times$ $10^{-7}|Z|^{-1}(E / \mathrm{TeV})$. Hence, taking a TeV upper limit for highest expected energy electrons, the corresponding upper limit on rigidity will be far outside the classical Böhm regime of diffusion ( $\rho \sim 1)$ within which particles execute a Brownian random walk with steps $\sim r_{\mathrm{g}}$ in a time $\sim 3 r_{\mathrm{g}} / c$.

As an alternative framework, in quasilinear theory the diffusion coefficients are calculated along the unperturbed, helical trajectory in a mean background magnetic field $\mathbf{B}_{0}$ [29]. The theory is valid so long as the energy in resonant waves is much smaller than that in the 


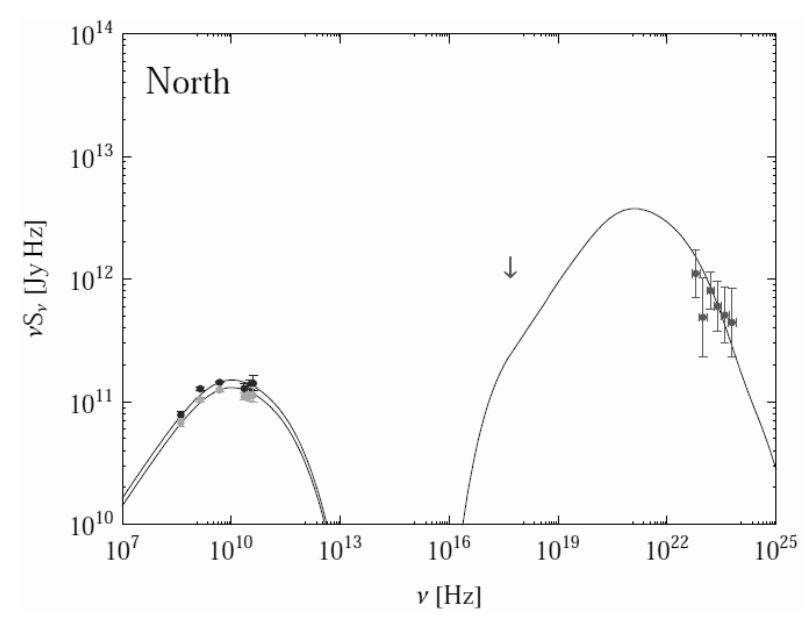

FIGURE 4. Northern GL of Cen A. Total inverse-Compton emission and radio measurements are presented as points with error bars and model for different regions is plotted as lines. for further information on model and data see [28]. From [28]. Reprinted with permission from AAAS.

background field and may be used to derive a diffusion coefficient for transport parallel to the mean magnetic field given by $\kappa_{\|} \approx 4.08 \mathrm{~L}_{\mathrm{c}} c \rho^{1 / 3}$. For the parameters under discussion this translates to an residence time $\tau_{\text {res }} \approx$ $L^{2} / 2 \kappa_{\|}=83(E / \mathrm{TeV})^{-1 / 3} \mathrm{Myr}$. Hence above a critical energy $\tau_{\text {crit }} \approx 2 \mathrm{GeV}$, electrons will cool too rapidly to permeate the lobes via quasi-linear diffusion. Given the evidence, it would appear that either electrons at the highest energies must be transported more effectively than permitted by quasi-linear theory, or some form of re-energizing must be occurring which compensates for rapid radiative losses. In the former case, a number of processes have been considered by Blundell and Rawlings [23]. In the case of pUHECR, the cooling times are well in excess of residence times within the lobes.

For the remainder of this paper, I shall focus on reviewing the state of play with regard to reacceleration of electrons and heavier particles within the GLs of Cen A through the second order Fermi process.

\section{STOCHASTIC ACCELERATION}

\section{Quasilinear theory}

A particle with velocity $\mathbf{v}_{1}$ and energy $E_{1}$ has an energy $E_{2}$ following an elastic scattering event with a scattering centre (magnetic field fluctuation) of velocity $\mathbf{U}$. The change in energy is given by $\Delta E \equiv E_{2}-E_{1}=$ $\left(2 \Gamma^{2} E_{1} / c^{2}\right)\left(\mathbf{U}-\mathbf{v}_{1}\right) \cdot \mathbf{U}$ where the Lorentz factor of the scattering centre is $\Gamma \equiv\left(1-U^{2} / c^{2}\right)^{-1 / 2}$. A particle can thus gain or lose energy depending on whether the collisions are head-on $\left(\mathbf{v}_{1} \cdot \mathbf{U}<1\right)$ or rear-end $\left(\mathbf{v}_{1} \cdot \mathbf{U}>1\right)$.
Second-order Fermi acceleration occurs through multiple scattering events which, on aggregate, result in a positive drift in particle energy. The evolution of the energetic particle distribution can be described by the momentum diffusion equation [30]

$$
\frac{\partial f(p, t)}{\partial t}=\frac{1}{p^{2}} \frac{\partial}{\partial p}\left[p^{2} D(p) \frac{\partial f(p, t)}{\partial p}\right] .
$$

The momentum diffusion coefficient $D(p)$ describing protons propagating in a cold static medium with lefthand and right-hand polarized Alfvén waves streaming with the same intensity parallel and anti-parallel to a mean field $\mathbf{B}_{0}$, may be written as [31]

$$
D(p)=d\left(\beta_{\mathrm{A}}\right)^{2}\left(\frac{r_{\mathrm{g}}}{\mathrm{L}_{\max }}\right)^{q-1} \frac{\delta B^{2}}{B_{0}^{2}} \frac{p^{2} c^{2}}{r_{\mathrm{g}} c}
$$

where $d$ is a numerical factor, $q$ is the index of the one-dimensional power spectrum $W(k) \propto k^{-q}$, where $\delta B^{2} / 8 \pi=\int_{k_{\max }}^{k_{\max }} W(k) \mathrm{d} k$ and $r_{g}=p c / Z e B$ is the gyroradius of a particle of charge number $Z$ (defined with respect to the root mean square magnetic field $B$ ), and $\beta_{\mathrm{A}}$ is the Alfvén velocity normalized to the speed of light. The associated acceleration timescale implicit in Equation 1 is $t_{\mathrm{acc}}=p^{2} / D(p)$. Assuming a pure power-law Kolmogorov spectrum with $q=5 / 3$ implies a maximum fluctuation scale of the field $\mathrm{L}_{\max }=(2 \pi / 0.77) \mathrm{L}_{\mathrm{c}}=$ $82 \mathrm{kpc}$ [32] and numerical factor in Equation 2 of order unity $(d=1.17)$. For a field with equal energy in the mean and turbulent components Equation 2 then yields

$$
\begin{aligned}
\left(\frac{t_{\mathrm{acc}}}{\mathrm{Myr}}\right) \approx & 10^{3}|Z|^{-\frac{1}{3}}\left(\frac{\mathrm{L}_{\mathrm{c}}}{10 \mathrm{kpc}}\right)^{\frac{2}{3}}\left(\frac{B}{1 v \mathrm{G}}\right)^{-\frac{7}{3}} \\
& \left(\frac{n_{\mathrm{p}}}{10^{-4} \mathrm{~cm}^{-3}}\right)\left(\frac{E}{1 \mathrm{TeV}}\right)^{\frac{1}{3}} .
\end{aligned}
$$

The validity of quasi-linear theory for conditions pertinent to the lobes of radio galaxies is confirmed by O'Sullivan et al. [33].

\section{Electron stochastic acceleration timescale}

Matching the timescales for the most energetic electrons by demanding $\tau_{\text {cool }}=\tau_{\text {acc }}$ places a constraint on the characteristic proton density within the lobe plasma [33]. For $\mathrm{L}_{\mathrm{c}}=10 \mathrm{kpc}$ requires $\left(n_{\mathrm{p}} / 10^{-4} \mathrm{~cm}^{-3}\right) \approx 1.1 \times 10^{-3}\left(E_{e} / \mathrm{TeV}\right)^{-4 / 3}(B / \mu \mathrm{G})^{\frac{7}{3}}$. The overall picture of the GLs of Cen A presented by observations from Fermi-LAT and earlier observations is that of an environment with $B \lesssim 1 \mu \mathrm{G}$ and $E_{e} \lesssim 1 \mathrm{TeV}$. Assuming these values for our analysis implies a characteristic proton density of $n_{\mathrm{p}}=10^{-7} \mathrm{~cm}^{-3}$. 
Considerations of the X-ray emission from the lobes [22] and Faraday rotation studies [34] indicate an upper bound for the thermal gas number density of $10^{-4} \mathrm{~cm}^{-3}$. This upper bound is three orders of magnitude higher than the value inferred above - either a stronger magnetic field or a lower maximum electron energy will reduce this difference.

\section{Stochastic acceleration of UHECR}

Equation 3 may be recast in the form $\left(t_{\mathrm{acc}} / \mathrm{Myr}\right) \approx$ $100|Z|^{-\frac{1}{3}}(B / 1 \mu \mathrm{G})^{-7 / 3}\left(n_{\mathrm{p}} / 10^{-7} \mathrm{~cm}^{-3}\right)(E / 1 \mathrm{EeV})^{1 / 3}$. Hence, it is feasible for the second-order Fermi acceleration to yield $\mathrm{EeV}$ protons if the baryon number density is three orders of magnitude lower than current observationally inferred upper estimates. For iron nuclei the corresponding limiting energy is $26 \mathrm{EeV}$. Therefore, without an extreme adjustment of currently accepted lobe conditions, stochastic acceleration is not a convincing mechanism for acceleration of UHECR to the very highest energies. It is possibly of greater interest as a generator of heavier nUHECR.

As a final comment on the acceleration of UHECR within the GLs of Cen A, the Hillas criterion [35] may be stated as a condition that the acceleration process must terminate once the gyroradius of the accelerating particle reaches the scale of the system. For the assumed conditions within this discussion, the limiting energies of UHECR are 100 times the maximal values derived above.

\section{CONCLUSIONS}

The properties of the GLs of Cen A have been reviewed in light of current observational data. The implications for transport and second-order Fermi acceleration are discussed.

\section{ACKNOWLEDGMENTS}

I am grateful to the organizers of UHECR2010 for their support and kind hospitality. I am indebted to Andrew Taylor and Brian Reville for invaluable discussions.

\section{REFERENCES}

1. J. Abraham, and et al., Physical Review Letters 101, 061101-+ (2008), 0806.4302.

2. J. Abraham, and et al., Physics Letters B 685, 239-246 (2010), 1002 . 1975.
3. R. U. Abbasi, and et al., Physical Review Letters 100, 101101-+ (2008), arXiv:astro-ph/0703099.

4. R. U. Abbasi, and et al., Astroparticle Physics 32, 53-60 (2009), 0904.4500.

5. J. Abraham, and et al., Science 318, 938- (2007), 0711.2256.

6. J. Abraham, and et al., ArXiv e-prints (2009), 0906.2347.

7. R. U. Abbasi, and et al., Astroparticle Physics 30, 175-179 (2008), 0804.0382.

8. D. Hooper, and A. M. Taylor, Astroparticle Physics 33, 151-159 (2010), 0910.1842.

9. J. Abraham, and et al., Physical Review Letters 104, 091101-+ (2010), 1002.0699.

10. R. U. Abbasi, and et al., ApJ 622, 910-926 (2005), arXiv:astro-ph/0407622.

11. R. U. Abbasi, and et al., Physical Review Letters 104, 161101-+ (2010), 0910.4184.

12. G. A. Medina Tanco, and et al., ApJ 492, 200-+ (1998), arXiv:astro-ph/9707041.

13. D. V. Semikoz, ArXiv e-prints (2010), 1009.3879.

14. G. Giacinti, and et al., J. Cosmology Astropart. Phys. 8, 36-+ (2010), 1006.5416.

15. J. O. Burns, E. D. Feigelson, and E. J. Schreier, ApJ 273, 128-153 (1983).

16. P. A. Hughes, Beams and Jets in Astrophysics, Cambridge University Press, 1991, URL http: //dx.doi.org/10.1017/CB09780511564703.

17. J. H. Croston, and et al., MNRAS 395, 1999-2012 (2009), 0901.1346.

18. R. P. Kraft, and et al., ApJ 698, 2036-2047 (2009), 0903.2663.

19. N. Junkes, and et al., A\&A 269, 29-38 (1993).

20. J. J. Condon, ARA\&A 30, 575-611 (1992).

21. F. A. Aharonian, MNRAS 332, 215-230 (2002), arXiv:astro-ph/0106037.

22. M. J. Hardcastle, and et al., MNRAS 393, 1041-1053 (2009), 0808.1593.

23. K. M. Blundell, and S. Rawlings, AJ 119, 1111-1122 (2000), arXiv:astro-ph/0001327.

24. C. L. Carilli, and et al., ApJ 383, 554-573 (1991).

25. A. G. Pacholczyk, Radio astrophysics. Nonthermal processes in galactic and extragalactic sources, Freeman, San Francisco, 1970.

26. N. S. Kardashev, Soviet Ast. 6, 317-+ (1962).

27. W. J. Jaffe, and G. C. Perola, A\&A 26, 423-+ (1973).

28. A. A. Abdo, and et al., Science 328, 725- (2010), 1006.3986.

29. P. Duffy, J. G. Kirk, Y. A. Gallant, and R. O. Dendy, A\&A 302, L21+ (1995), arXiv:astro-ph/9509058.

30. B. A. Tverskoi, Soviet Journal of Experimental and Theoretical Physics 25, 317-+ (1967).

31. R. Schlickeiser, ApJ 336, 243-293 (1989).

32. F. Casse, M. Lemoine, and G. Pelletier, Phys. Rev. D 65, 023002-+ (2002), arXiv:astro-ph/ 0109223.

33. S. O'Sullivan, B. Reville, and A. M. Taylor, MNRAS 400 , 248-257 (2009), 0903.1259.

34. I. J. Feain, and et al., ApJ 707, 114-125 (2009), 0910.3458.

35. A. M. Hillas, ARA\&A 22, 425-444 (1984). 